\title{
Relaciones tróficas entre mamíferos herbívoros nativos y exóticos del Parque Provincial Ischigualasto (San Juan, Argentina)
}

\author{
María L. Reus ${ }^{1, \otimes}$; Claudia de los Ríos ; Begoña Peco ${ }^{4}$; Stella M. Giannoni ${ }^{12,3}$ \\ \& Claudia M. Campos ${ }^{5}$ \\ ${ }^{1}$ Departamento de Biología, Facultad Ciencias Exactas, Físicas y Naturales (FCEFN), Universidad Nacional de San Juan, \\ Argentina (UNSJ). ${ }^{2}$ Centro de Investigación de la Geósfera y la Biósfera (CIGEOBIO), CONICET-UNSJ. ${ }^{3}$ Instituto y Museo \\ de Ciencias Naturales (IMCN), FCEFN-UNSJ. ${ }^{4}$ Departamento de Ecología, Universidad Autónoma de Madrid, España. \\ ${ }^{5}$ Instituto Argentino de Investigaciones de las Zonas Áridas (Universidad Nacional de Cuyo-Gobierno de Mendoza- \\ CONICET), Argentina.
}

\begin{abstract}
Resumen. Cada vez es más frecuente que las comunidades de herbívoros estén compuestas por especies nativas y exóticas. Las especies exóticas, con una historia reciente de coexistencia entre ellas y con las especies nativas, presentarían menos estrategias de reparto de recursos que las especies nativas, que co-evolucionaron juntas y establecieron relaciones entre sí y su ambiente. Con el fin de evaluar las relaciones tróficas en una comunidad de herbívoros que coexisten en el Parque Provincial Ischigualasto se analizaron las composiciones estacionales de las dietas, las amplitudes de los nichos tróficos y los solapamientos dietarios, y se realizaron asociaciones en base a la condición (nativo o exótico) y a los tamaños corporales (grandes o pequeños). En la región hiperárida del Monte, donde se desarrolló el estudio, los arbustos tuvieron la cobertura más representativa a lo largo del año. Las dietas de los herbívoros nativos y exóticos estuvieron basadas en este grupo funcional de plantas. En particular, las especies nativas mantuvieron un uso intenso y sostenido de los arbustos durante las estaciones húmeda y seca. Las especies exóticas presentaron mayor similitud entre sus dietas y amplitudes de nicho trófico constantes a lo largo del año (y más amplias que las amplitudes de nicho de las especies nativas). Las dietas de las especies nativas presentaron menor similitud entre ellas y los nichos tróficos fueron más estrechos, especialmente en la estación seca. La superposición de dieta entre nativos y exóticos no varió entre las estaciones ni siquiera considerando especies de tamaños corporales similares. Es probable que no se hayan detectado superposiciones importantes en el uso de los recursos tróficos debido a las densidades bajas de especies exóticas presentes en la actualidad en el Parque Provincial Ischigualasto. De todas maneras, se recomienda un monitoreo permanente que permita evaluar potencial competencia entre especies nativas y exóticas.
\end{abstract}

[Palabras clave: burro, Desierto del Monte, dietas, guanaco, liebre europea, mara, rata vizcacha, vaca]

\begin{abstract}
Trophic interactions among native and exotic herbivorous mammals in the Ischigualasto Provincial Park (San Juan, Argentina). The communities of herbivores are more frequently composed of native and exotic species. Exotic species, with a recent history of co-occurrence among them and with native species, would exhibit less resource distribution strategies than native species, which co-evolved together establishing relationships between them and their environment. Aiming to assess trophic relationships in a community of herbivores that co-occur in Ischigualasto Provincial Park, we analyzed seasonal diet compositions, trophic niche breadth and diet overlap, and made associations based on condition (native or exotic) and body size (large or small). In the hyper-arid Monte region, where the study was conducted, shrub cover was the most representative cover throughout the year. The diets of native and exotic herbivores were based on this functional group of plants. Particularly native species made intensive and sustained use of shrubs during both the wet and dry seasons. Exotic species showed higher similarity in their diets and a constant trophic niche breadth all over the year (broader than that of native species). There was less similarity among the diets of native species, and their trophic niche was narrower, particularly in the dry season. Diet overlap between natives and exotics showed no variations between seasons, not even considering species of similar body size. Probably, no important overlaps are detected in food resource use because of the low densities of exotic species currently present in Ischigualasto Provincial Park. In any event, we recommend permanent monitoring that allows assessing potential competition between native and exotic species.
\end{abstract}

[Keywords: cow, diets, donkey, guanaco, European hare, mara, Monte Desert, viscacha rat]

Editora asociada: María Semmartin

$\triangle$ reusster@gmail.com 


\section{INTRODUCCIÓN}

Los estudios clásicos de ecología trófica de ungulados, en particular rumiantes, sentaron las bases teóricas que intentan explicar el uso de recursos alimentarios por los mamíferos herbívoros (Illius and Gordon 1992; Gordon and Illius 1994; 1996; Codron et al. 2007). En general, se reconocen tres estrategias tróficas fundamentales que se basan sobre adaptaciones anatómicas y fisiológicas de los animales, más allá del tamaño corporal: el pastoreo, el ramoneo y el consumo mixto (Hofmann 1984, 1989). Los pastoreadores son herbívoros eficientes en la absorción de las partes solubles de las células vegetales, en particular de gramíneas, con un paso rápido del alimento no digestible (Hofmann 1989; Gordon and Illius 1996). Los ramoneadores, en cambio, consumen principalmente dicotiledóneas y realizan una fermentación intensa del alimento, para lo que cuentan con un rumen largo que permite un mayor tiempo de retención de las partes no digestibles (Hofmann 1989). Los consumidores mixtos tienen una morfología digestiva intermedia, con características propias de los pastoreadores y ramoneadores, por lo que pueden alternar entre el consumo de dicotiledóneas y gramíneas (Hofmann 1989). En este continuo de pastoreo-ramoneo fueron agregándose otras categorías como frugivoría y frugivoría/ ramoneo (Bodmer 1990) o pastoreo obligado y facultativo (Gagnon and Chew 2000).

Mientras que algunos trabajos como los recién citados se centran en las adaptaciones fisiológicas a los distintos tipos de dieta (modelos relacionados con el tipo de dieta; e.g., Hofmann 1989), otros autores enfatizan las relaciones entre el tamaño corporal, los requerimientos energéticos y la habilidad en el procesado del alimento (modelos relacionados con la calidad del alimento; e.g., Gordon and Illius 1996). En este último caso, el tamaño de los animales, la calidad del forraje y las relaciones filogenéticas entre los herbívoros cobran importancia para explicar el uso de los recursos alimentarios por ungulados (Jarman 1974; Gordon and Illius 1994; Clauss et al. 2003; Gordon 2003). Se propone, entonces, que los herbívoros grandes pueden sobrevivir con dietas de baja calidad, pero requieren una tasa alta de ingesta de alimento, en contraposición con los herbívoros pequeños, que necesitan dietas de alta calidad y bajo volumen de alimento (Jarman 1974; Demment and Van Soest 1985). Al combinar ambos modelos (tipo de dieta y calidad del alimento) se sugirió que los herbívoros grandes, capaces de utilizar dietas de baja calidad, consumen más gramíneas que las especies pequeñas.

A nivel global, cada vez es más frecuente la coexistencia de herbívoros nativos y exóticos en los ecosistemas, una situación que también es común en las tierras secas de la Argentina. En estos ambientes, los herbívoros deben enfrentar presiones selectivas relacionadas con la escasez de agua, las temperaturas extremas, la baja productividad primaria y la disponibilidad de plantas arbustivas con componentes anti-herbívoro (Ojeda and Tabeni 2009). Con el fin de evaluar las relaciones tróficas en un ensamble de mamíferos herbívoros y considerando que las dietas de los herbívoros del Monte muestran cambios estacionales en su composición (Campos 1997; Reus 2015; Reus et al. 2013, 2014), el presente estudio tiene como objetivos: 1) analizar las asociaciones entre las dietas de las diferentes especies en las estaciones seca y húmeda, y también explorar la existencia de asociaciones según el origen de las especies (nativo o exótico) y los tamaños corporales (grandes o pequeños), y 2) comparar los grupos funcionales de plantas que constituyen las dietas, las amplitudes de los nichos tróficos y los solapamientos dietarios entre los herbívoros, considerando el origen y los tamaños corporales.

Se espera encontrar que las especies exóticas, con una historia reciente de coexistencia entre sí y con las especies nativas, presenten menos estrategias de reparto de recursos que las especies nativas, que co-evolucionaron juntas estableciendo relaciones entre sí y su ambiente (Kirchoff and Larsen 1998; Kelley et al. 2002; Madhusudan 2004). Si se considera que las especies nativas usan de manera diferencial los recursos tróficos, ya que evolucionaron relacionadas entre sí y con las plantas del ambiente (Bailey and Schweitzer 2010), se espera encontrar una similitud baja entre las dietas de los herbívoros nativos.

En base a las diferencias en el tamaño corporal de los herbívoros, se predicen mayores similitudes entre las dietas de especies con tamaños corporales semejantes. Además, las especies más pequeñas serán más selectivas en su dieta (menores amplitudes de nicho trófico) que los herbívoros grandes, que pueden utilizar recursos abundantes de baja calidad nutricional (Jarman 1974). De acuerdo con la hipótesis de la calidad selectiva (Jarman and Sinclair 1979), se predice que los herbívoros 
nativos serán menos selectivos durante la estación húmeda, cuando la abundancia de recursos aumenta debido a la presencia de mayores cantidades de alimentos de alta calidad distribuidos de forma homogénea. Entonces, las especies nativas presentarían mayores amplitudes de nicho trófico durante la estación húmeda. En cambio, cuando los recursos son pobres en cantidad y calidad, las dietas de los animales estarán compuestas por un grupo básico de plantas y los nichos tróficos serán estrechos. Por otro lado, asumiendo la introducción más reciente de los herbívoros exóticos, se espera encontrar un menor ajuste de las dietas a la vegetación disponible (Bailey and Schweitzer 2010), con nichos tróficos amplios a lo largo del año (dietas generalistas y oportunistas) y similitudes altas entre las dietas de las especies exóticas. Sumado a esto, en época de escasez de recursos (invierno o estación seca), los efectos de la competencia aumentan ya que las condiciones ambientales son limitantes (Prins et al. 2006; Shrader et al. 2006). Por lo tanto, la superposición entre las dietas de especies nativas y exóticas debería ser mayor, en especial entre especies de tamaño corporal similar (Wiens 1993).

\section{Materiales y MÉTOdOS}

\section{Sitio de estudio y cuantificación de la oferta de recursos alimentarios}

El trabajo se desarrolló en el Parque Provincial Ischigualasto (PPI), que ocupa una superficie de 62916 ha al noreste de la provincia de San Juan (3005' S - 6755' O; altitud media 1300 m s.n.m.), departamento de Valle Fértil. El PPI se encuentra en la porción más árida del Monte y está incluido principalmente en el Monte de Sierras y Bolsones (Burkart et al. 1999). El clima es árido, con una precipitación media anual de $183 \mathrm{~mm}$. Se caracteriza por tener precipitaciones estivales (de noviembre a marzo, la precipitación media es $100 \mathrm{~mm}$ ) e inviernos secos (de abril a octubre, la precipitación media es $8 \mathrm{~mm}$ ). La temperatura media en verano es $24.3^{\circ} \mathrm{C}$, con una máxima absoluta de $45^{\circ} \mathrm{C}$. La temperatura media en invierno es $8.5^{\circ} \mathrm{C}$, con un mínimo absoluto de $-10^{\circ} \mathrm{C}$ (De Fina et al. 1962).

En los años 2005 y 2006 se tomaron los datos de disponibilidad de recursos tróficos y se recolectaron las muestras de heces; se abarcó una estación seca (abril a diciembre) y una estación húmeda (noviembre a marzo). El trabajo se llevó a cabo a lo largo de tres comunidades vegetales descriptas para el PPI: el chical, las laderas con cactus columnares y el algarrobal (Márquez et al. 2005; Acebes et al. 2010). Se seleccionaron estas comunidades porque en ellas se encontró evidencia de actividad reciente de mamíferos herbívoros nativos y exóticos, determinada por la presencia de huellas, heces, cuevas y signos de ramoneo.

Como un estimador de la disponibilidad de alimento para los herbívoros se registró la cobertura vegetal específica en las comunidades. Las coberturas se cuantificaron en parcelas de $50 \mathrm{~m}^{2}$ seleccionadas aleatoriamente y separadas entre sí por al menos $30 \mathrm{~m}$, con un total de 45 parcelas en la estación húmeda y 33 parcelas en la estación seca. En cada una de las parcelas se establecieron dos transectas de $25 \mathrm{~m}$ cada una, perpendiculares entre sí. Sobre las transectas se estimó la cobertura vegetal en 13 cuadrados de $2 \mathrm{~m}^{2}$, a través de la proyección del área expuesta de cada planta sobre el suelo. El primer cuadrado se localizó en la intersección de las transectas y el resto de los cuadrados se establecieron cada $2 \mathrm{~m}$ sobre las transectas.

\section{Especies y análisis de dietas}

En el presente trabajo se estudiaron tres especiesnativascon problemas deconservación (Ojeda et al. 2012): la rata vizcacha Octomys mimax (Rodentia: Octodontidae), la mara Dolichotis patagonum (Rodentia: Caviidae) y el guanaco Lama guanicoe (Cetartiodactyla: Camelidae), y tres especies exóticas: el burro Equus africanus asinus (Perissodactyla: Equidae), la vaca Bos taurus (Artiodactyla: Bovidae) y la liebre europea Lepus europaeus (Lagomorpha: Leporidae). La rata vizcacha, la mara y la liebre europea fueron consideradas herbívoros de pequeño tamaño corporal. Las especies que superan los $15 \mathrm{~kg}$, como el guanaco, el burro y la vaca, fueron considerados herbívoros grandes. Todas estas especies coexisten en los sitios del PPI donde se realizaron los muestreos, y si bien los datos sobre sus densidades dentro del PPI son escasos, se estima, por ejemplo, que la población actual de guanacos es de 330 individuos (F. Cappa, comunicación personal), mientras que la cantidad de vacas varía según el manejo de ganado que realizan los pobladores aledaños.

Se recolectaron las heces frescas de los herbívoros dentro de cada parcela. Las heces se identifican fácilmente en el campo por sus características de tamaño, forma y color. Las muestras de heces de guanaco se obtuvieron 
a partir de los "bosteaderos comunales", y se extrajeron entre 15 y 25 unidades por muestra a partir de diferentes bosteaderos separados por al menos $20 \mathrm{~m}$. Las muestras de heces de burro y vaca pesaron $\sim 50 \mathrm{~g}$, y se obtuvieron a partir de diferentes deposiciones distanciadas al menos $20 \mathrm{~m}$. Para los casos de liebre europea y mara, las muestras consistieron en un conjunto de cinco a 10 pellets por cada deposición encontrada, separadas por al menos $20 \mathrm{~m}$. Las muestras de heces de rata vizcacha estuvieron conformadas por todos los excrementos nuevos recolectados en las cuevas que presentaban actividad reciente evidenciada por la presencia de cúmulos frescos de vegetación.

La composición botánica de la dieta se determinó mediante el análisis microhistológico de heces según la técnica de Dacar y Giannoni (2001). Se sistematizó la observación de tres preparados por muestra de heces; en cada uno se observaron 50 campos microscópicos bajo aumento de 400x. Se identificaron los distintos ítems alimentarios a nivel de género o especie cuando fue posible, y se determinó la frecuencia relativa de abundancia de cada ítem en la dieta (Holechek and Gross 1982).

\section{Análisis estadísticos}

Asociaciones entre las dietas de los herbívoros para cada estación. Para evaluar la asociación en base a la similitud de las matrices de datos de las composiciones de las dietas de los diferentes herbívoros durante las estaciones húmeda y seca se realizaron análisis de redundancia (RDA), un análisis de ordenación restringida que utiliza variables explicativas nominales. Los efectos de las variables se evaluaron a través de permutaciones de Monte Carlo (Ter Braak and Smilauer 2002). Luego de evaluar las asociaciones estacionales se exploró el efecto del origen de las especies (nativo o exótico) y el tamaño corporal (grande o pequeño) como variables explicativas. Los análisis se aplicaron a las matrices de datos y se incluyeron sólo aquellas especies vegetales que aparecían en al menos cinco muestras. Los resultados fueron visualizados en diagramas de ordenación. El poder explicativo de los modelos obtenidos se evaluó a través del porcentaje de variabilidad explicada por los tres primeros ejes. Los análisis y figuras se realizaron usando el lenguaje $R$ 3.3.1 ( $R$ Development Core Team 2016).

Comparaciones entre las dietas. Las especies vegetales que componen la oferta de recursos alimentarios y las dietas de los herbívoros fueron clasificadas dentro de los siguientes grupos funcionales: árboles, arbustos, herbáceas anuales, gramíneas, cactus y bromeliáceas. Para realizar las comparaciones estacionales entre las dietas y la disponibilidad de recursos tróficos considerando los grupos funcionales se utilizó el análisis de varianza no paramétrico Kruskal-Wallis y comparaciones a posteriori con el método Holm-Bonferroni $(P<0.05)$ (InfoStat 2008).

Se estimó el índice de diversidad ecológica de Shannon-Weaver (Colwell and Futuyma 1971) como un indicador de la amplitud de nicho trófico, es decir, una medida de la riqueza y la abundancia de especies de plantas en las dietas $\left(\mathrm{H}^{\prime}=-\sum \mathrm{p}_{\mathrm{j}}^{*} \log \mathrm{p}_{\mathrm{j}^{\prime}}\right.$ donde $\mathrm{p}_{\mathrm{j}}=\mathrm{n}_{\mathrm{i}} / \mathrm{N}$ es la proporción del número total de plantas de la especie $\mathrm{j}, \mathrm{n}_{\mathrm{i}}=$ número de plantas de la especie $\mathrm{j}$ y $\mathrm{N}=$ número total de plantas). Para detectar diferencias entre las amplitudes de nichos tróficos de las especies considerando su origen (nativo y exótico) y tamaño corporal (grandes y pequeñas) se utilizó la prueba de Mann-Whitney-Wilcoxon.

Con el fin de estimar el solapamiento dietario, considerando todos los ítems identificados, se aplicó el índice de Pianka (Pianka 1986): $\mathrm{O}=\left(\sum \mathrm{p}_{\mathrm{ij}}^{*} \mathrm{p}_{\mathrm{ik}}\right) /\left(\left(\mathrm{p}_{\mathrm{ij}}^{2} \sum \mathrm{p}_{\mathrm{kj}}{ }^{2}\right)^{1 / 2}\right)$, donde $\mathrm{O}$ es el grado de solapamiento entre la especie $\mathrm{j}$ y $\mathrm{k}$, y $p_{i j}$ y $p_{i k}$ son las proporciones del recurso $i$ utilizado por la especie j y $k$, respectivamente. Los valores de solapamiento entre pares de especies oscilan entre cero y uno, donde cero indica que no hay solapamiento y uno indica una superposición completa. La prueba de Mann-Whitney-Wilcoxon permitió comparar el solapamiento dietario entre especies nativas y exóticas durante las estaciones húmeda y seca y el solapamiento entre especies nativas y exóticas de tamaños corporales similares en ambas estaciones.

\section{Resultados}

\section{Asociaciones entre las dietas de los herbivoros para cada estación}

Debido a que muchas especies de gramíneas no pudieron ser determinadas, las mismas fueron reunidas en un único ítem. Durante la estación seca, las dietas de las distintas especies mostraron asociaciones significativas $\left(R^{2}=0.515 ;\right.$ adj. $\left.R^{2}=0.475 ; F_{5}=12.767 ; P<0.001\right)$ y los primeros tres ejes explicaron $28.84 \%$, $12.29 \%$ y $5.60 \%$ del total de la variabilidad, respectivamente (Figura 1). Las dietas del guanaco, el burro y la vaca fueron similares 
entre sí y estuvieron muy asociadas al consumo de gramíneas, de arbustos como Zuccagnia punctata (Fabaceae), Trichomaria usillo (Malpighiaceae), Cyclolepis genistoides (Asteraceae), Larrea spp. (Zygophyllaceae), la bromelia Tillandsia sp. (Bromeliaceae) y el árbol Prosopis chilensis (Fabaceae). La dieta de la rata vizcacha se relacionó con el arbusto Capparis atamisquea (Capparaceae) y el cactus Teprhocactus sp. (Cactaceae), la dieta de la mara con $P$. torquata (Fabaceae), y la de la liebre europea con Bulnesia retama (Zygophyllaceae), Atriplex sp. (Chenopodiaceae) y Ephedra sp. (Ephedraceae) (Figura 1; Tablas 1 y 2). Para esta misma estación, el tamaño corporal de las especies mostró tener mayor poder explicativo de las asociaciones que la condición nativo o exótico de los herbívoros $\left(\mathrm{R}^{2}=0.252\right.$; adj. $\mathrm{R}^{2}=0.240 ; \mathrm{F}_{5}=21.636 ; P<0.005 ;$ los tres primeros ejes explicaron $25.3 \%$, $24.1 \%$ y $12.2 \%$ del total de la variabilidad).

Durante la estación húmeda, las dietas de las especies también mostraron asociaciones significativas $\left(\mathrm{R}^{2}=0.416\right.$; adj. $\mathrm{R}^{2}=0.377$; $\left.\mathrm{F}_{5}=10.703 ; P<0.001\right)$ y los tres primeros ejes explicaron $27.48 \%, 6.62 \%$ y $3.97 \%$ del total de la variabilidad (Figura 2). Las dietas del burro, la vaca y la liebre europea fueron similares y estuvieron asociadas a gramíneas,

Tabla 1. Composición de la dieta (media del porcentaje relativo de ocurrencia de las especies vegetales \pm error estándar) de las especies nativas: rata vizcacha (Octomys mimax), mara (Dolichotis patagonum) y guanaco (Lama guanicoe) durante las estaciones húmeda y seca en el Parque Provincial Ischigualasto (San Juan, Argentina). Sólo las plantas con porcentajes $\geq 1 \%$ fueron incluidas; $n$ es el número de muestras de heces. Se listan los acrónimos (Acr) de las especies que aparecen en el RDA. Cuando se presenta el porcentaje total del grupo funcional, todas las especies que aparecieron en la oferta $\mathrm{y}$ en las dietas fueron consideradas.

Table 1. Composition of diets (mean $\pm \mathrm{SE}$ of relative percentage of occurrence of plant species) of native species: viscacha rat (Octomys mimax), mara (Dolichotis patagonum) and guanaco (Lama guanicoe) during the wet and the dry seasons in the Ischigualasto Provincial Park (San Juan, Argentina). Only plant species with percentages $\geq 1 \%$ were included; $\mathrm{n}$ is the number of faecal samples. The acronym (Acr) of the species used in redundancy analysis (RDA) (Figures 1 and 2) is given

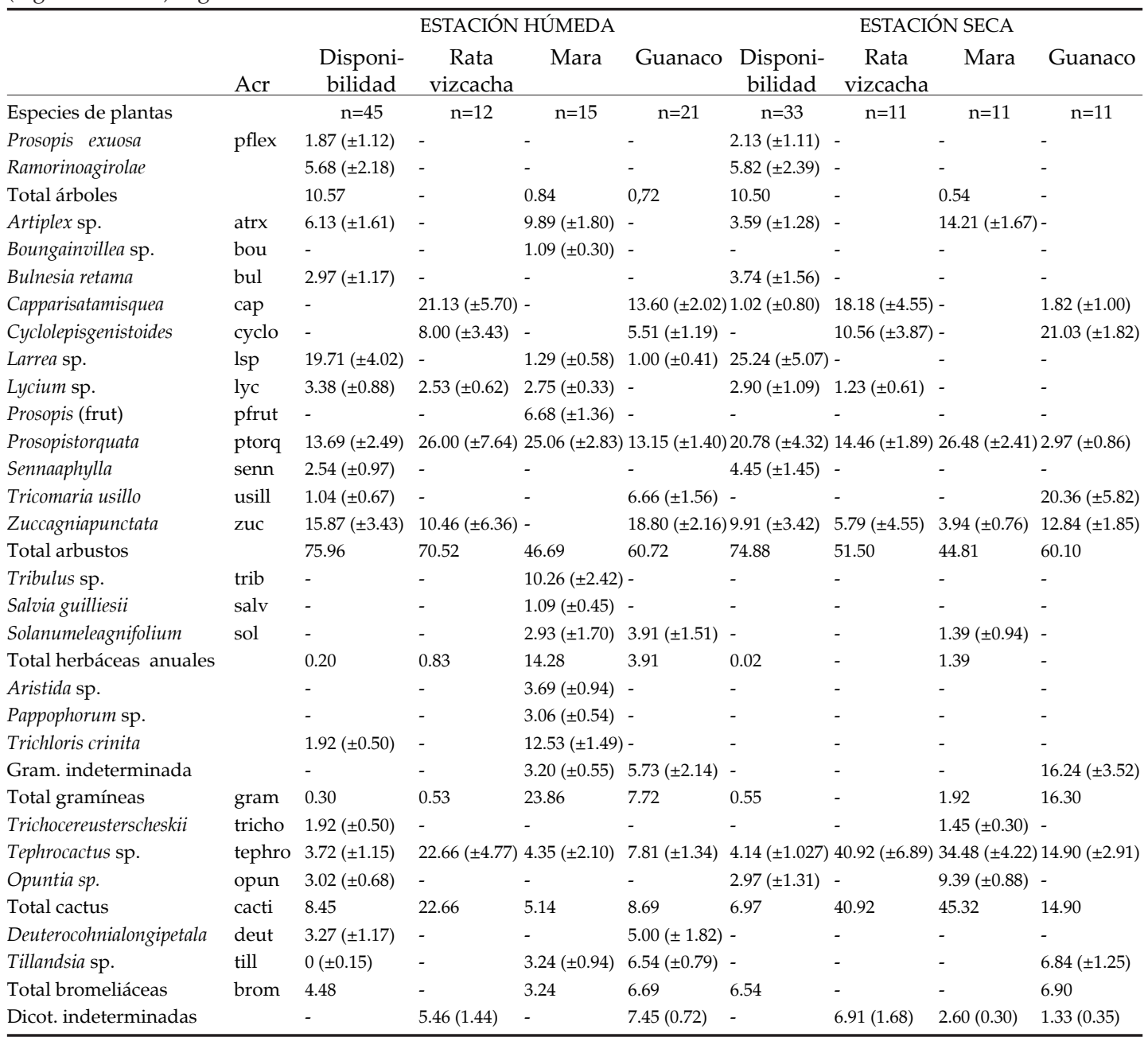




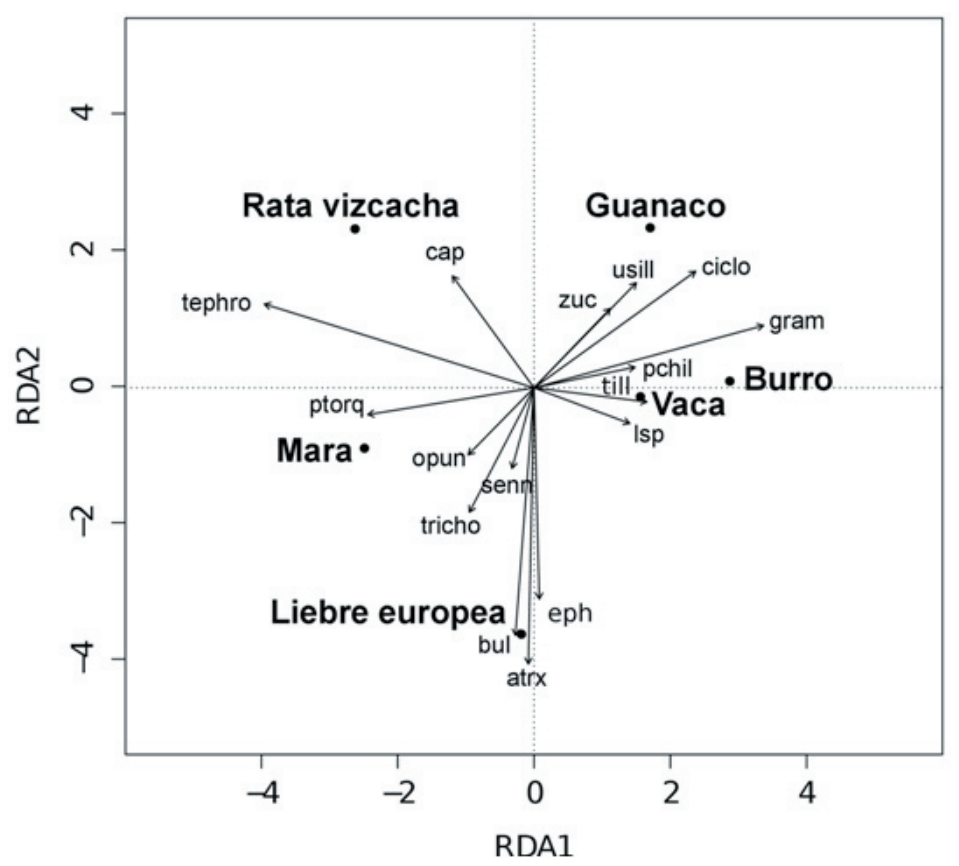

Figura 1. Biplot del análisis de redundancia (RDA) de las especies de plantas en las dietas y las especies de herbívoros como variables explicativas, durante la estación seca. Los acrónimos de las especies se indican en las Tablas 1 y 2. En el gráfico las especies de plantas en las dietas se representan con flechas y las variables explicativas de indican con puntos. Las flechas que apuntan hacia un punto de una variable explicativa indican una alta correlación positiva, las flechas que apuntan en dirección opuesta indican una alta correlación negativa. Las especies con flechas de mayor longitud y las variables más alejadas del centro son las más importantes en el análisis.

Figure 1. Redundancy analysis (RDA) biplot of plant species in diets of dry season using herbivorous species as explanatory parameters. For abbreviations of plant species see tables 1 and 2 . In the biplot plant species in the diets were represented by arrows and explanatory parameters were indicated by points. Arrows pointing towards an explanatory parameter point indicate a high positive correlation, arrows pointing in an opposite direction indicate a high negative correlation and arrows pointing at a right angle from a line connecting the environmental variable point with the center indicate a near-zero correlation. Species with longer arrows and environmental variable points further from the center are more important in the analysis.

a Deuterocohnia longipetala (Bromeliaceae) y a C. genistoides. La mara y la rata vizcacha compartieron la asociación con $P$. torquata, mientras que la dieta del guanaco se relacionó con arbustos como Z. punctata, C. atamisquea, Boungainvillea sp. (Nyctaginaceae) y T. usillo (Figura 2; Tablas 1 y 2). Para la estación húmeda, el carácter nativo o exótico de las especies es la variable que mejor explica las asociaciones entre las dietas de los herbívoros $\left(\mathrm{R}^{2}=0.189 ;\right.$ adj. $\mathrm{R}^{2}=0.179 ; \mathrm{F}_{5}=18.454 ; P<0.005 ; \mathrm{los}$ tres primeros ejes explicaron $18.9 \%, 26.3 \%$ y $15.3 \%$ del total de la variabilidad).

\section{Comparaciones entre las dietas}

La cobertura vegetal media en el ambiente fue $17.65 \%$ (error estándar=8.86) durante la estación seca (32 especies vegetales; índice de Shannon=2.41) y $19.37 \%$ (error estándar=6.73) durante la estación húmeda (44 especies; índice de Shannon=2.71). Las especies vegetales más representativas en la oferta a lo largo del año fueron los arbustos Larrea sp., Z. punctata, $P$. torquata y Atriplex sp., y la bromeliácea $D$. longipetala (Tabla 1 y 2). Los arbustos fueron el tipo de cobertura más abundante, seguida por los cactus y los árboles en la estación húmeda y por los cactus, las bromeliáceas y los árboles en la estación seca (Tabla 3). La cobertura de las gramíneas y las herbáceas anuales fue muy baja todo el año, no superando el 1\% (Tablas 1 y 2).

La composición de las dietas de los herbívoros se presenta en las Tablas 1 y 2 . La contribución de los diferentes grupos funcionales en las dietas de los herbívoros varió significativamente en cada estación, aunque los arbustos siempre fueron uno de los grupos más representativos (Tabla 1, 2 y 3). Las dietas de las especies nativas estuvieron compuestas principalmente por arbustos durante la estación húmeda y por arbustos acompañados por cactus en la estación seca. Las especies exóticas también consumieron altos porcentajes de arbustos, aunque incorporaron herbáceas anuales y gramíneas (Tabla 1, 2 y 3). Las especies de menor tamaño corporal consumieron arbustos, 
Tabla 2. Composición de la dieta (media del porcentaje relativo de ocurrencia de las especies vegetales \pm error estándar) de las especies exóticas: liebre europea (Lepus europaeus), burro (Equus africanus asinus) y vaca (Bos taurus) durante las estaciones húmeda y seca en el Parque Provincial Ischigualasto (San Juan, Argentina). Sólo las plantas con porcentajes $\geq 1 \%$ fueron incluidas; $n$ es el número de muestras de heces. Se listan los acrónimos (Acr) de las especies que aparecen en el RDA. Cuando se presenta el porcentaje total del grupo funcional, todas las especies que aparecieron en la oferta y en las dietas fueron consideradas.

Table 2. Composition of diets (mean \pm SE of relative percentage of occurrence of plant species) of exotic species: European hare (Lepus europaeus), donkey (Equus africanus asinus), and cow (Bos taurus) during the wet and the dry seasons in the Ischigualasto Provincial Park (San Juan, Argentina). Only plant species with percentages $\geq 1 \%$ were included; $\mathrm{n}$ is the number of faecal samples. The acronym (Acr) of the species used in redundancy analysis (RDA) (Figures 1 and 2) is given.

\begin{tabular}{|c|c|c|c|c|c|c|c|}
\hline & \multirow[b]{2}{*}{ Acr } & \multicolumn{3}{|c|}{ ESTACIÓN HÚMEDA } & \multicolumn{3}{|c|}{ ESTACIÓN SECA } \\
\hline & & Liebre Europea & Burro & Vaca & Liebre europea & Burro & Vaca \\
\hline Especies de plantas & & $\mathrm{n}=16$ & $\mathrm{n}=11$ & $\mathrm{n}=8$ & $\mathrm{n}=13$ & $\mathrm{n}=13$ & $\mathrm{n}=5$ \\
\hline Prosopis flexuosa & pflex & - & - & - & - & $1.23( \pm 0.70)$ & - \\
\hline Prosopischilensis & pchil & - & $7.94( \pm 3.65)$ & - & - & $1.23( \pm 0.43)$ & - \\
\hline Geoffroeadecorticans & geof & $3.31( \pm 0.91)$ & - & - & $1.91( \pm 0.81)$ & - & - \\
\hline Total árboles & & 3.31 & 8.18 & - & 1.91 & 1.46 & - \\
\hline Artiplex sp. & atrx & $6.58( \pm 1.18)$ & $8.42( \pm 1.74)$ & $7.90( \pm 3.22)$ & $21.33( \pm 4.17)$ & $7.28( \pm 1.75)$ & $9.66( \pm 1.99)$ \\
\hline Bulnesia retama & bul & $1.33( \pm 0.52)$ & - & - & $19.28( \pm 2.40)$ & - & - \\
\hline Capparisatamisquea & cap & - & $2.06( \pm 1.29)$ & - & - & - & - \\
\hline Cyclolepisgenistoides & cyclo & $19.58( \pm 3.92)$ & $14.12( \pm 3.17)$ & $6.85( \pm 2.01)$ & - & $14.55( \pm 3.04)$ & $16.66( \pm 3.11)$ \\
\hline Ephedra sp. & eph & $1.74( \pm 0.62)$ & - & - & - & - & - \\
\hline Larrea sp. & lsp & - & $1.33( \pm 0.31)$ & $0.95( \pm 0.54)$ & $1.13( \pm 0.91)$ & $3.32( \pm 1.54)$ & - \\
\hline Lycium sp. & lyc & $4.45( \pm 0.77)$ & - & $3.14( \pm 1.86)$ & $1.59( \pm 0.81)$ & $1.02( \pm 0.31)$ & - \\
\hline Prosopis (frut) & pfrut & $8.45( \pm 1.35)$ & $1.45( \pm 1.45)$ & $12.09( \pm 2.90)$ & - & - & - \\
\hline Prosopistorquata & ptorq & $8.75( \pm 2.95)$ & $7.15( \pm 1.68)$ & $7.42( \pm 2.92)$ & $7.28( \pm 2.04)$ & $4.64( \pm 2.58)$ & - \\
\hline Sennaaphylla & senn & - & - & - & $2.20( \pm 0.75)$ & - & - \\
\hline Tricomaria usillo & usill & - & - & - & - & $3.08( \pm 1.17)$ & - \\
\hline Zuccagniapunctata & zuc & - & $3.94( \pm 0.77)$ & $1.52( \pm 1.31)$ & - & $4.18( \pm 0.88)$ & $11.87( \pm 2.19)$ \\
\hline Total arbustos & & 50.30 & 40.52 & 40.74 & 53.94 & 37.99 & 42.71 \\
\hline Hyalisargentea & hyal & - & - & $2.38( \pm 1.52)$ & - & - & $2.53(1.77)$ \\
\hline Tribulus sp. & trib & $9.66( \pm 2.11)$ & - & - & - & - & - \\
\hline Solanumeleagnifolium & sol & $1.12( \pm 0.55)$ & - & - & $2.19( \pm 1.19)$ & - & - \\
\hline Total herbáceas anuales & & 11.57 & 2.76 & 2.38 & 2.19 & 2.66 & 2.53 \\
\hline Aristida sp. & & $3.91( \pm 1.07)$ & $4.12( \pm 1.77)$ & $2.29( \pm 0.79)$ & - & $7.70( \pm 3.35)$ & - \\
\hline Cortaderia sp. & & - & - & $1.05( \pm 0.95)$ & - & - & - \\
\hline Cotteapappaphoroides & & $6.21( \pm 1.87)$ & - & - & - & - & - \\
\hline Distichilisspicata & & $1.25( \pm 0.43)$ & $14.00( \pm 3.99)$ & $7.33( \pm 1.73)$ & - & $6.19( \pm 1.43)$ & $4.26( \pm 2.19)$ \\
\hline Pappophorum sp. & & $1.00( \pm 0.33)$ & $5.03( \pm 1.44)$ & $4.85( \pm 2.29)$ & - & $4.44( \pm 1.88)$ & $2.13( \pm 1.22)$ \\
\hline Sporobolusrigens & & $3.37( \pm 1.47)$ & - & $5.90( \pm 4.01)$ & - & $1.95( \pm 1.34)$ & - \\
\hline Trichloris crinita & & $6.75( \pm 1.28)$ & $14.78( \pm 3.34)$ & $17.71( \pm 3.27)$ & - & $9.77( \pm 2.14)$ & $6.40( \pm 2.82)$ \\
\hline Gram. indeterminada & & $1.50( \pm 0.30)$ & $2.06( \pm 1.05)$ & $2.66( \pm 0.52)$ & - & - & $1.86( \pm 0.57)$ \\
\hline Total gramíneas & gram & 23.99 & 42.17 & 41.89 & 0.82 & 33.53 & 15.73 \\
\hline Trichocereusterscheskii & tricho & - & - & - & $3.69( \pm 0.90)$ & - & - \\
\hline Tephrocactus sp. & tephro & $3.50( \pm 1.20)$ & - & $3.05( \pm 1.52)$ & $15.18( \pm 3.96)$ & $1.55( \pm 0.83)$ & $10.93( \pm 3.21)$ \\
\hline Opuntiasp. & opun & - & - & - & $1.02( \pm 0.40)$ & - & $12.13( \pm 3.27)$ \\
\hline Total cactus & cacti & 4.25 & 1.39 & 3.81 & 19.89 & 2.78 & 23.33 \\
\hline Deuterocohnialongipetala & deut & - & $5.27( \pm 3.62)$ & - & - & - & $0.80( \pm 0.80)$ \\
\hline Tillandsia sp. & till & $4.46( \pm 0.87)$ & - & $4.95( \pm 3.28)$ & $5.38( \pm 1.21)$ & $3.73( \pm 1.07)$ & $13.60( \pm 3.32)$ \\
\hline Total bromeliáceas & brom & 4.46 & 5.94 & 5.61 & 5.38 & 4.08 & 14.40 \\
\hline Dicot. indeterminada & & $2.58(0.68)$ & $2.24(0.58)$ & $1.14(0.43)$ & $1.84(0.26)$ & $2.27(0.68)$ & - \\
\hline
\end{tabular}

cactus, gramíneas y herbáceas, mientras que las especies más grandes además de arbustos incorporaron gramíneas, particularmente el burro y la vaca (Tabla 3).

A lo largo del año los herbívoros nativos presentaron un nicho trófico más estrecho que los exóticos (prueba de Mann-WhitneyWilcoxon, $W=3727.5 ; P<0.0001$; amplitud de nicho de especies exóticas $=2.10$; de especies nativas $=1.73$ ). Estacionalmente, las especies nativas y exóticas respondieron de forma diferente en relación a las amplitudes de sus nichos tróficos. Las especies nativas redujeron la amplitud del nicho trófico durante la estación seca, mientras que las exóticas no cambiaron las amplitudes tróficas estacionalmente (especies nativas: amplitud de nicho en estación seca=1.53; en estación húmeda $=1.91, \mathrm{~W}=2843.5 ; P<0.0001$; especies exóticas: en estación seca $=2.07$; en estación húmeda=2.13). En general, las especies más pequeñas tuvieron un nicho trófico más estrecho que las grandes $(\mathrm{W}=3071$; 
Tabla 3. Composición de las dietas de los herbívoros en base a los grupos funcionales de plantas. Se muestran los grupos funcionales que aparecen como más representativos en primer y segundo lugar según la prueba de Kruskal-Wallis y la prueba de comparaciones a posteriori siguiendo el método Holm-Bonferroni. ${ }^{* * * *}$ indica $P<0.0001, * * P<0.01$; / precede el segundo grupo más representativo; + indica que no hay diferencia significativa entre esos grupos.

Table 3. Composition of herbivores' diets based on plant functional groups. The first and second more representative groups are shown (Kruskal-Wallis test and post hoc Holm-Bonferroni method). ${ }^{* * *} P<0.0001,{ }^{* *} P<0.01$; / precedes the second more representative group; + indicates no significant difference among groups.

\begin{tabular}{lllllll}
\hline & \multicolumn{4}{c}{ ESTACIÓN HÚMEDA } & \multicolumn{2}{c}{ ESTACIÓN SECA } \\
& $\mathrm{Chi}^{2}$ & $P$ & Grupos de plantas & $\mathrm{Chi}^{2}$ & $P$ & Grupos de plantas \\
\hline Disponibilidad & 169.33 & $* * * *$ & arbus/cactus+árbol & 111.40 & $* * * *$ & arbus/cactus+brom+árbol \\
Rata vizcacha & 53.84 & $* * * *$ & arbus/cactus & 68.75 & $* * * *$ & arbus+cactus \\
Mara & 63.03 & $* * * *$ & arbus/gram+herb & 50.64 & $* * * *$ & arbus+cactus \\
Guanaco & 87.13 & $* * * *$ & arbus & 56.86 & $* * * *$ & arbus \\
Liebre europea & 64.21 & $* * * *$ & arbus/gram+herb & 51.70 & $* * * *$ & arbus/cactus \\
Burro & 43.35 & $* * * *$ & arbus+gram & 43.28 & $* * * *$ & arbus+gram \\
Vaca & 29.49 & $* * * *$ & arbus+gram & 20.21 & $* *$ & arbus \\
\hline
\end{tabular}

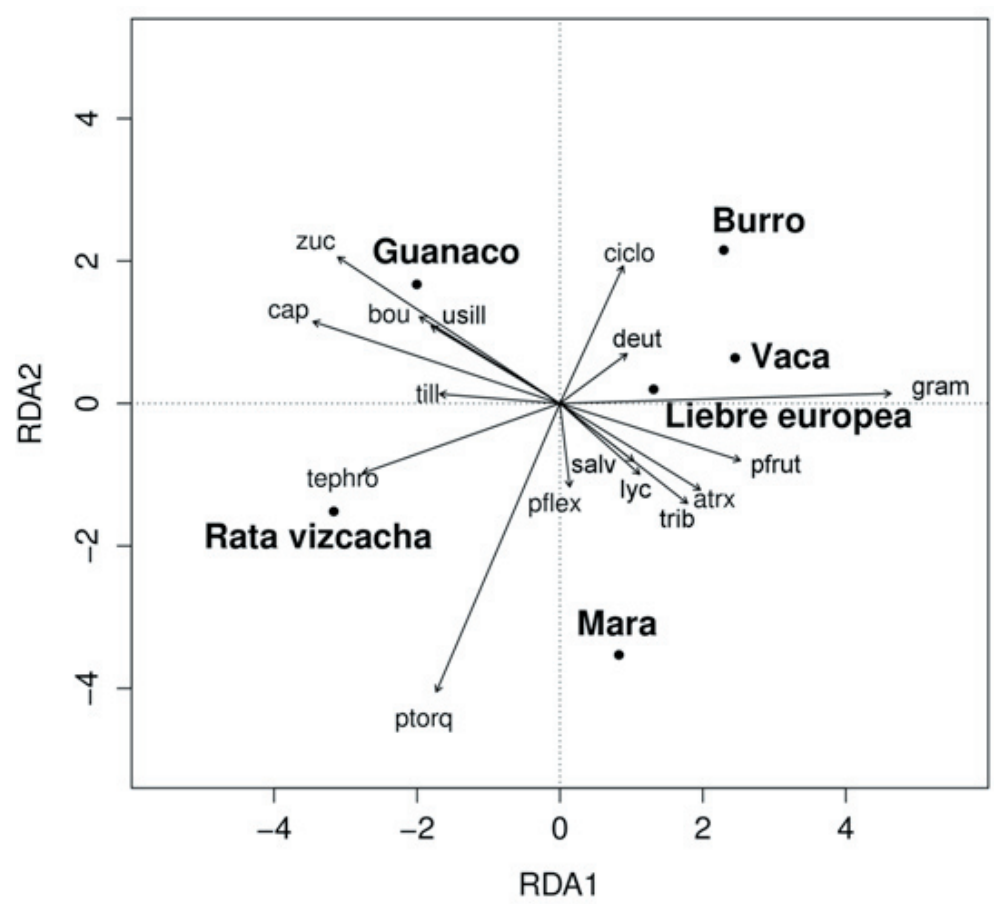

Figura 2. Biplot del análisis de redundancia (RDA) de las especies de plantas en las dietas y las especies de herbívoros como variables explicativas, durante la estación húmeda. Los acrónimos de las especies se indican en las Tablas 1 y 2. En el gráfico las especies de plantas en las dietas se representan con flechas y las variables explicativas de indican con puntos. Las flechas que apuntan hacia un punto de una variable explicativa indican una alta correlación positiva, las flechas que apuntan en dirección opuesta indican una alta correlación negativa. Las especies con flechas de mayor longitud y las variables más alejadas del centro son las más importantes en el análisis.

Figure 2. Redundancy analysis (RDA) biplot of plant species in diets of wet season using herbivorous species as explanatory parameters. For abbreviations of plant species see tables 1 and 2 . In the biplot plant species in the diets were represented by arrows and explanatory parameters were indicated by points. Arrows pointing towards an explanatory parameter point indicate a high positive correlation, arrows pointing in an opposite direction indicate a high negative correlation, and arrows pointing at a right angle from a line connecting the environmental variable point with the center indicate a near-zero correlation. Species with longer arrows and environmental variable points further from the center are more important in the analysis.

$P<0.01$; especies grandes $=2.03$; especies pequeñas=1.80). Los nativos pequeños tuvieron menores amplitudes de nicho que los nativos de mayor tamaño corporal ( $\mathrm{W}=876$; $P<0.01$; especies grandes $=1.90$; especies pequeñas $=1.64$ ). En cambio, las especies exóticas no presentaron cambios en las amplitudes de nicho considerando el tamaño corporal (especies grandes=2.14; especies pequeñas=2.05).

Finalmente, la superposición de los nichos tróficos entre las especies nativas y exóticas fue similar en ambas estaciones (estación seca $=0.32$; estación húmeda $=0.24$ ). Si bien la prueba de Mann-Whitney-Wilcoxon no 
detectó diferencias significativas, se observa una superposición de nichos más alta en la estación seca entre herbívoros nativos y exóticos de tamaños similares (superposición entre nativos y exóticos grandes en la estación seca $=0.33$; entre nativos y exóticos pequeños $=0.54$; entre nativos y exóticos grandes en la estación húmeda $=0.16$; entre nativos y exóticos pequeños $=0.27$ ).

\section{DisCUSIÓN}

En el área de estudio, la cobertura de plantas nunca pasó el 20\%. Los arbustos tuvieron la cobertura más representativa a lo largo del año y las dietas de los herbívoros estuvieron basadas en este grupo de plantas, en especial en las especies nativas. Como se esperaba encontrar, las especies nativas tuvieron baja similitud entre sus dietas y mostraron, entonces, segregación en el uso de recursos tróficos. Incluso las diferencias se acentuaron en la estación seca, cuando el guanaco aumentó el consumo de gramíneas y su dieta se asemejó más a las dietas de los herbívoros exóticos de tamaño corporal grande. En cambio, las dietas de las especies exóticas fueron similares en términos globales, y compartieron el consumo de gramíneas principalmente en la estación húmeda. En cuanto a las amplitudes de los nichos tróficos, los herbívoros nativos presentaron amplitudes menores que los exóticos, amplitudes que se redujeron aun más durante la estación seca y en el caso de los herbívoros nativos de menor tamaño corporal. En cambio, las superposiciones de los nichos tróficos entre nativos y exóticos no variaron entre estaciones ni al considerar los tamaños corporales de las especies.

Seguramente, en ambientes áridos extremos, como Ischigualasto, una buena estrategia para los mamíferos herbívoros es consumir el recurso alimentario más estable y abundante a lo largo del año (e.g., los arbustos). Los arbustos proveen forraje verde a lo largo del año, aun bajo condiciones ambientales críticas (Sala et al. 1989; Papachristou et al. 2005). Si bien algunos arbustos abundantes en la oferta ambiental son pobremente palatables (como las especies de Larrea) (Guevara and Grunwald 2012), y otros muestran diferencias entre hojas nuevas y maduras en las concentraciones de nutrientes (como L. divaricata y A. lampa) (Carrera et al. 2000), en general, los arbustos del Monte presentan pocas variaciones intraanuales porque pueden completar sus ciclos fenológicos de forma independiente de las precipitaciones (Cavagnaro and Passera 1991). Los herbívoros nativos mostraron una respuesta trófica plástica y flexible, ajustada a este ambiente en particular, ya que sus dietas estuvieron constituidas por una base fuerte de arbustos. Este grupo no es el principal componente de las dietas de estas especies en otros ecosistemas. Por ejemplo, la dieta de poblaciones de guanaco en ecosistemas de la Patagonia está compuesta principalmente por gramíneas y dicotiledóneas herbáceas, mientras que las arbustivas fueron evitadas (Raedeke 1980; Bonino and Pelliza Sbriller 1991; Puig et al. 1996, 1997, 2011; Baldi et al. 2004). En el presente estudio, como también se encontró en el ecotono entre Monte y Chaco seco en la provincia de San Luis (Gómez Vinassa and Núñez 2016), el guanaco no mostró un consumo alto de gramíneas. De hecho, los arbustos fueron el ítem más importante, mientras que los cactus y las gramíneas fueron incorporados sobre todo en la estación seca ( 30\% de la dieta).

En cuanto al más pequeño de los herbívoros nativos, la rata vizcacha, los cúmulos que almacena en las cuevas donde vive presentan restos decactus, ramas de Halophytumameghinoi (Halophytaceae) y frutos de Ramorinoa girolae (Fabaceae) (Campos et al. 2015). Estos últimos frutos son leñosos y duros, y la rata vizcacha los perfora para extraer las semillas. También remueve semillas de Prosopis a partir de los excrementos de burro y mara (Sobrero et al. 2010). Los resultados del presente trabajo mostraron que la dieta de la rata vizcacha incluyó los arbustos $P$. torquata y $C$. atamisquea. Este último contiene grandes cantidades de resinas y taninos (Sassi et al. 2011). Siguiendo la definición de Shipley y colaboradores (2009) se puede considerar a la rata vizcacha como un especialista facultativo porque tiene la habilidad de detoxificar componentes químicos específicos y consumir plantas difíciles (como los cactus, C. atamisquea y las semillas de $R$. girolae), sin sacrificar la opción de incorporar otras especies de plantas en su dieta. En épocas de escasos recursos, puede evitar la competencia con herbívoros más generalistas y plásticos, como el guanaco y la mara, porque la rata vizcacha es capaz de explotar recursos que son poco utilizado por los otros herbívoros.

En Ischigualasto, las gramíneas tienen abundancia muy baja y distribución restringida. El consumo de alimentos ricos en fibras, como las gramíneas, es difícil para los herbívoros tanto por su baja digestibilidad como por la limitada entrada de nutrientes (Cork 1994; Carrera et al. 2000). En este trabajo se observó que las gramíneas fueron 
consumidas principalmente por los herbívoros grandes, sobre todo exóticos, mientras que los más pequeños las incorporaron en cantidades bajas (mara y liebre europea) o muy bajas (rata vizcacha) durante la estación húmeda, cuando las gramíneas más comunes del Monte tienen la mayor digestibilidad y los contenidos más altos de nutrientes y energía (ver Guevara et al. 2009 y referencias citadas). En comparación con los herbívoros pequeños, se sabe que los herbívoros grandes presentan tasas más altas de retención del alimento y mayor capacidad digestiva, características que facilitan la digestión y absorción de fibras (Hume 1989; Batzli and Hume 1994).

En un ensamble de especies nativas se puede esperar que exista segregación en el uso de los recursos tróficos porque las especies evolucionaron en simpatría, con un reparto en la explotación de los alimentos (Jarman and Sinclair 1979). En el caso de las especies nativas de Ischigualasto, si bien consumieron altas cantidades de arbustos, sus dietas no fueron similares porque globalmente incorporaron distintas especies de plantas. En cambio, las especies exóticas presentaron una similitud elevada entre sus dietas a lo largo del año. Los herbívoros exóticos compartieron la asociación con las gramíneas, en particular durante la estación seca. Por ejemplo, la dieta de la vaca estuvo compuesta cerca de $40 \%$ por arbustos, como en otras áreas del Monte, pero las gramíneas estuvieron presentes en porcentajes importantes todo el año (Guevara et al. 2010; Guevara and Grunwald 2012).

Nuestros resultados acuerdan con la hipótesis de la calidad selectiva (Jarman and Sinclair 1979), ya que los herbívoros nativos tienen nichos tróficos más estrechos que las especies exóticas, en especial durante la estación seca, hecho que se ve acentuado en el caso de los herbívoros más pequeños. Estos resultados podrían implicar que los nativos incluyen pocos ítems, pero usan las plantas más nutritivas del ambiente, probablemente haciendo frente a las defensas químicas de los arbustos que crecen en el Monte. El herbívoro nativo más pequeño, la rata vizcacha, es la especie que tiene el nicho trófico más estrecho.

Finalmente, en relación a la superposición en el uso de los recursos tróficos, varios estudios previos realizados en una comunidad vegetal específica dentro del PPI e involucrando algunas de las especies consideradas en el presente trabajo muestran que la superposición entre las dietas de la liebre europea y la mara aumenta en la estación seca (Reus et al. 2013), así como la superposición entre las dietas del guanaco y del burro (Reus et al. 2014). Al realizar comparaciones más globales, que incorporan más especies y comunidades vegetales, en el presente estudio no se logró detectar diferencias en la superposición de uso de los recursos tróficos. Es difícil establecer cuándo un recurso es limitante (De Boer and Prins 1990) y probablemente en ambientes áridos las limitaciones en la disponibilidad de recursos alimentarios se relacionen más con la calidad de los alimentos que con la cantidad. Sumado a esto, la superposición en las dietas puede verse desdibujada porque los animales, según su capacidad de desplazamiento, se mueven buscando alimento en los sitios donde estos están disponibles (Prins 2000).

Tanto los estudios previos acerca del uso de hábitat (Ovejero et al. 2011; Acebes et al. 2012) y de recursos alimentarios (Reus et al. 2013, 2014) por herbívoros nativos y exóticos del PPI como los resultados del presente trabajo muestran que en las densidades actuales de especies exóticas no se manifiestan superposiciones significativas con las nativas en el uso de los recursos. Por otro lado, tanto los herbívoros nativos como los exóticos están cumpliendo roles ecológicos clave en la dispersión de semillas de algarrobo (Prosopis spp.) (Campos et al. 2008, 2016). Sin embargo, se propone el monitoreo continuo de las poblaciones para detectar cualquier cambio que pudiera afectar de alguna manera a las especies nativas protegidas en el PPI.

Agradecimientos. La investigación fue financiada por el Proyecto INTERMARG (Conservación Biológica de la Fundación BBVA). Los autores argentinos son investigadores de CONICET y docentes de la Universidad Nacional de San Juan. Damos las gracias al personal del Parque Provincial Ischigualasto por su apoyo. Las muestras se procesaron en el Laboratorio de microhistología del Instituto y Museo de Ciencias Naturales (Universidad Nacional de San Juan). Este estudio fue parte de la tesis doctoral de la primera autora en el PROBIOL (Universidad Nacional de Cuyo).

\section{REFERENCIAS}

Acebes, P., J. Traba, B. Peco, M. L. Reus, S. M. Giannoni, and J. E. Malo. 2010. Abiotic gradients drive floristic composition and structure of plant communities in the Monte Desert. Rev Chil Hist Nat 83:395-407.

Acebes, P., J. Traba, and J. E. Malo. 2012. Co-occurrence and potential for competition between wild and domestic 
large herbivores in a South American desert. J Arid Environ 77:39-44.

Bailey, J. K., and J. A. Schweitzer. 2010. The role of plant resistance and tolerance to herbivory in mediating the effects of introduced herbivores. Biol Invas 12:337-51.

Baldi, R., A. Pelliza Sbriller, D. Elston, and S. Albon. 2004. High potential for competition between guanacos and sheep in Patagonia. J Wildl Manage 68:924-38.

Batzli, G., and I. Hume. 1994. Foraging and digestion in herbivores. Pp. 313-314 in D. J. Chivers and P. Langer (eds.). The digestive system in mammals. Food form and function. Cambridge University Press, Cambridge, United Kingdom.

Bodmer, R. E. 1990. Ungulate frugivores and the browser-grazer continuum. Oikos 57:319-325.

Bonino, N., and A. Pelliza Sbriller. 1991. Composición botánica de la dieta de guanaco (Lama guanicoe) en dos ambientes contrastantes en Tierra del Fuego, Argentina. Ecol Austral 1:97-102.

Burkart, R., N. O. Barbaro, R. O. Sánchez, and D. A. Gómez. 1999. Ecorregiones de la Argentina. Administración de Parques Nacionales, Buenos Aires, Argentina.

Campos, C. M. 1997. Utilización de recursos alimentarios por mamíferos medianos y pequeños del Desierto del Monte. Tesis doctoral. Facultad de Ciencias Exactas, Físicas y Naturales, Universidad Nacional de Córdoba, Argentina. Pp. 185.

Campos, C. M., B. Peco, V. E. Campos, J. E. Malo, S. M. Giannoni, and F. Suárez. 2008. Endozoochory by native and exotic herbivores in dry areas: consequences for germination and survival of Prosopis seeds. Seed Sci Res 18:91-100.

Campos, C. M., V. E. Campos, F. Miguel, and M. I. Cona. 2016. Management of protected areas and its effect on an ecosystem function: removal of Prosopis exuosa seeds by mammals in Argentinian drylands. PLoS One 11(9):e0162551. doi:10.1371/journal.pone.0162551

Campos, V. E., S. Giannoni, L. Reus, G. Díaz, and C. Campos. 2015. Is availability of trophic resources related to crevices selected by Octomys mimax in the monte desert? Mastozool Neotrop 22:279-287.

Carrera, A. L., C. L. Sain, and M. B. Bertiller. 2000. Patterns of nitrogen conservation in shrubs and grasses in the Patagonian Monte, Argentina. Plant Soil 224:185-193.

Cavagnaro, J. B., and C. B. Passera. 1991. Water utilization by shrubs and grasses in the Monte ecosystem, Argentina. Pp. 255-258 in A. Gaston., M. Kernick, H. N. Le Houerou (eds.). Proceedings of the Four International Rangeland Congress, CIRAD (SCIST), Montpellier, Francia.

Clauss, M., M. Lechner-Doll, and W. J. Streich. 2003. Ruminant diversification as an adaptation to the physicomechanical characteristics of forage. A reevaluation of an old debate and a new hypothesis. Oikos 102:253-262.

Codron, D., J. A. Lee-Thorp, M. Sponheimer, J. Codron, D. De RuiterII and J. S. Brink.2007. Significance of diet type and diet quality for ecological diversity of African ungulates. J Anim Ecol 76:526-537.

Colwell, R. K., and D. J. Futuyma. 1971. On the measurement of niche breadth and overlap. Ecology 52:567-576.

Cork, S. J. 1994. Digestive constraints on dietary scope in small and moderately-small mammals: how much do we really understand? Pp. 337-369 in D. J. Chivers and P. Langer (eds.). The digestive system in mammals. Food form and function. Cambridge University Press, Cambridge, United Kingdom.

Dacar, M., and S. M. Giannoni. 2001. Technical note: A simple method for preparing reference slides of seed. J Range Manage 54:191-193.

De Boer, W. F., and H. H. T. Prins. 1990. Large herbivores that strive mightily but eat and drink as friends. Oecologia 82:264-274.

De Fina, A. L., F. Giannetto, and L. J. Sabella. 1962. Difusión geográfica de cultivos índices en la provincia de San Juan y sus causas. Publicación $N^{\circ}$ 80. Instituto de Suelos y Agrotecnia e INTA, Buenos Aires.

Demment, M. W., and P. J. Van Soest. 1985. A nutritional explanation for body-size patterns of ruminant and nonruminant herbivores. Am Nat 125:641-672.

Gagnon, M., and A. E. Chew. 2000. Dietary preferences in extant African Bovidae. J Mammal 81:490-511.

Gómez Vinassa, M. L., and M. B. Núñez. 2016. Dieta estacional de guanacos (Lama guanicoe) y burros ferales (Equus asinus) en un ambiente semiárido de San Luis, Argentina. Ecol Austral 26:178-188.

Gordon, I. J. 2003. Browsing and grazing ruminants: are they different beasts? Forest Ecol Manage 181:13-21.

Gordon, I. J., and A. W. Illius. 1994. The functional significance of the browser-grazer dichotomy in African ruminants. Oecologia 98:167-175.

Gordon, I. J., and A. W. Illius 1996. The nutritional ecology of African ruminants: a reinterpretation. J Anim Ecol 65: 18-28.

Guevara, J. C., E. G. Grünwaldt, O. R. Estévez, A. J. Bisigato, L. J. Blanco, F. N. Biurrun, C. A. Ferrando, C. C. Chirino, E. Morici, B. Fernández., L. I. Allegretti, and C. B. Passera. 2009. Range and livestock production in the Monte Desert, Argentina. J Arid Environ 73:228-237.

Guevara, J. C., L. I. Allegretti, O. R Estévez, A. S. Monge, J. A. Páez, and M. A. Cony. 2010. Diets of Domestic and Wild herbivores grazing in common in a Rangeland of Mendoza Province, Argentina. Pp. 463-477 in B. Veress. and J. Szigethy. (eds.). Horizons in Earth Science Research. Volume I. Nova Science Publishers. New York, USA.

Guevara, J. C., and E. G. Grunwaldt. 2012. The desert environment of Mendoza, Argentina: status and prospects for sustainable beef cattle production. Pp. 115-127 in J. C. Guevara, E. Grunwaldt and C. Sivaperuman (eds.). Deserts: Fauna, Flora and Environment. Nova Science Publishers. New York, USA.

Hofmann, R. R. 1984. Feeding habits of mouflon (Ovis ammon musimon) and chamois (Rupicapra rupicapra) in relation to the morphophysiological adaptation of their digestive tract. Pp. 341-355 in M. Hoefs (ed.). Northern wild sheep and goat council. Whitehorse, Yukon, Canadá.

Hofmann, R. R.1989. Evolutionary steps of ecophysiological adaptation and diversification of ruminants: a comparative 
view of their digestive system. Oecologia 78:443-457.

Holechek, J. L., and B. D. Gross. 1982. Evaluation of different calculation procedures for microhistological analysis. J Range Manage 35:721-730.

Hume, I. D. 1989. Optimal digestive strategies in mammalian herbivores. Physiol Zool 62:1145-1163.

Illius, A. W., and I. J. Gordon. 1992. Modelling the nutritional ecology of ungulate herbivores: evolution of body size and competitive interactions. Oecologia 89:428-434.

InfoStat. 2008. Software professional software version 2008. Grupo InfoStat, Facultad de Ciencias Exactas, Físicas y Naturales, Universidad Nacional de Córdoba, Argentina.

Jarman, P. J. 1974. The social organisation of antelope in relation to their ecology. Behaviour 48:215-267.

Jarman, P. J., and A. R. E. Sinclair. 1979. Feeding strategy and pattern of resource partitioning in ungulates. Pp. 130160 in A.R.E. Sinclair and M. Norton-Griffiths (eds.). Serengeti: dynamics of an ecosystem. University of Chicago Press, Chicago.

Kelley, M., R. Stewart, T. Bowyer, J. G. Kie, N. J. Cimon, and B. K. Johnsone. 2002. Temporospatial distributions of elk, mule deer, and cattle: Resource partitioning and competitive displacement. J Mamm 83:229-244.

Kirchoff, M. D., and D. N. Larsen. 1998. Dietary overlap between native sitka black-tailed deer and introduced elk in Southeast Alaska. J Wildl Manage 62:236-242.

Madhusudan, M. D. 2004. Recovery of wild large herbivores following livestock decline in a tropical Indian wildlife reserve. J Appl Ecol 41:858-869.

Márquez, J., E. Martínez Carretero, A. Dalmasso, G. Pastrán, and S. Ortiz. 2005. Las áreas protegidas de la provincia de San Juan (Argentina) II. La vegetación del Parque Provincial de Ischigualasto. Multequina 14:1-27.

Ojeda, R. A., and S. Tabeni. 2009. The mammals of the Monte Desert revisited. J Arid Environ 73:173-181.

Ojeda, R. A., V. Chillo, and G. B. Díaz Isenrath. 2012. Libro Rojo de mamíferos amenazados de la Argentina. Sociedad Argentina para el Estudio de los Mamíferos.

Ovejero, R. J. A., P. Acebes., J. E. Malo, J. Traba., M. E Mosca Torres., and C. E. Borghi. 2011. Lack of feral livestock interference with native guanaco during the dry season in a South American desert. Eur J Wildl Res 57:1007-1015.

Papachristou, T. G., L. E. Dziba, and F. D. Provenza. 2005. Foraging ecology of goats and sheep on wooded rangelands. Small Ruminant Research 59:141-156.

Pianka, E. R. 1986. Ecology and Natural history of desert Lizards. Analyses of the ecological niche structure and community. New Jersey: Princeton University Press. Pittsburg, Pennsylvania.

Prins, H. H. T., W. F. de Boer, H. Van Oeveren, A. Correia, J. Mafuca, and H. Olff. 2006. Co-existence and diet segregation of three small bovid species in Southern Mozambique. Afr J Ecol 44:186-198.

Prins, H. H. T. 2000. Competition between wildlife and livestock. Pp. 51-80 in H. H. T. Prins, J. G. Grootenhuis and T. T. Dolan (eds.). Wildlife conservation by sustainable use. Kluwer Academic Publishers, Boston, USA.

Puig, S., F. Videla, S. Monge, and V. Roig. 1996. Seasonal variations in guanaco diet (Lama guanicoe Müller 1776) and food availability in Northern Patagonia, Argentina. J Arid Environ 34:215-224.

Puig, S., F. Videla, and M. I. Cona. 1997. Diet and abundance of the guanaco (Lama guanicoe Müller 1776) in four habitats of northern Patagonia, Argentina. J Arid Environ 36:343-357.

Puig, S., M. I. Rosi, F. Videla, and E. Méndez. 2011. Summer and winter diet of the guanaco and food availability for a High Andean migratory population (Mendoza, Argentina). Mamm Biol 76:727-734.

Raedeke, K. 1980. Food habits of the guanaco (Lama guanicoe) of Tierra del Fuego, Chile. Turrialba 30:177-181.

Reus, M. L. 2015. Interacciones entre mamíferos nativos y exóticos y efecto sobre la vegetación, Reserva Provincial Ischigualasto, San Juan (Argentina). Tesis doctoral. Programa de Biología, Universidad Nacional de Cuyo, Mendoza, Argentina.

Reus, M. L., B. Peco., C. de los Ríos, S. M. Giannoni, and C. M. Campos. 2013. Trophic interactions between two medium-sized mammals: the case of the native Dolichotis patagonum and the exotic Lepus europaeus in a hyper-arid ecosystem. Acta Theriol 58:205-214.

Reus. M. L., F. Cappa, N. Andino, V. E. Campos, C. de los Ríos, and C. M. Campos. 2014. Trophic interactions between the native guanaco (Lama guanicoe) and the exotic donkey (Equus asinus) in the hyper-arid Monte desert (Ischigualasto Park, Argentina). Stud Neotrop Fauna E 49:159-168.

R Core Team. 2016. R: a language and environment for statistical computing. [Internet]. R foundation for statistical computing. Viena, Austria. URL: http:/ /www.r-project.org/

Sala, O. E., R. A. Golluscio, W. K. Lauenroth, and A. Soriano. 1989. Resource partitioning between shrubs and grasses in the Patagonian steppe. Oecologia 81:501-505.

Sassi, P. L., C. E. Borghi, M. A. Dacar, and F. Bozinovic. 2011. Geographic and seasonal variability in feeding behavior of a small herbivorous rodent. Acta Theriol 56:35-43.

Shipley, L. A., J. S. Forbey, and B. D. Moore. 2009. Revisiting the dietary niche: when is a mammalian herbivore a specialist? Integr Comp Biol 49:274-290.

Shrader, A. M., N. Owen-Smith, and J. O. Ogutu. 2006. How a mega-grazer copes with the dry season: nutrient intake rates by white rhinoceros in the wild. Funct Ecol 20:376-384.

Sobrero, R., V. E. Campos, S. M. Giannoni, and L. A. Ebensperger. 2010. Octomys mimax (Rodentia: Octodontidae). Mamm Spec 42(853):49-57.

Ter Braak, C. J. F., and P. Smilauer. 2002. CANOCO Reference Manual and CanoDraw for Windows User's Guide: Software for Canonical Community Ordination (version 4.5) Microcomputer Power, Ithaca, New York, USA.

Wiens, J. A. 1993. Fat times, lean times and competition among predators. Trends Ecol Evol 8:348-349. 